\title{
Signal Extraction and Separation in In Vivo Animal PET Studies with Masked Volumewise Principal-Component Analysis
}

\author{
Fredrik Engbrant ${ }^{1}$, Azita Monazzam ${ }^{1}$, Per-Edvin Svensson ${ }^{1}$, Johan Olsson ${ }^{1}$, Ewert Bengtsson ${ }^{2}$, and Pasha Razifar ${ }^{1}$ \\ ${ }^{I}$ Uppsala Applied Science Laboratory (UASL), GE Healthcare, Uppsala, Sweden; and ${ }^{2}$ Centre for Image Analysis, Uppsala University, \\ Uppsala, Sweden
}

\begin{abstract}
The standardized uptake value is commonly used as a tool to supplement visual interpretation and to quantify the images acquired from static in vivo animal PET. The preferred approach for analyzing PET data is either to sum the images and calculate the standardized uptake value or to use kinetic modeling. The aim of this study was to investigate the performance of masked volumewise principal-component analysis (MVW-PCA) used in dynamic in vivo animal PET studies to extract and separate signals with different kinetic behaviors. Methods: PET data were acquired with a small-animal PET scanner and a fluorine tracer in a study of rats and mice. After acquisition, the data were reconstructed by use of 4 time protocols with different frame lengths. Data were analyzed by use of MVW-PCA with applied noise prenormalization and a new masking technique developed in this study. Results: The resulting principal-component images showed a clear separation of the activity in the spine into the first MVW-PCA component and the activity in the kidneys into the second MVW-PCA component. In addition, the different time protocols were shown to have little or no impact on the results obtained with MVW-PCA. Conclusion: MVW-PCA can efficiently separate different kinetic behaviors into different principal-component images. Moreover, MVW-PCA is a stable technique in the sense that the time protocol chosen has only a small impact on the resulting principal-component images.
\end{abstract}

Key Words: masked volumewise principal-component analysis; PET; animal study; dynamic dataset; list mode

J Nucl Med Technol 2010; 38:53-60

DOI: $10.2967 /$ jnmt.110.075085

\section{$\mathbf{P}$} ET is a noninvasive imaging modality that is used to visualize the concentration of a molecule labeled with a radioactive isotope called a tracer, representing the physiologic interaction between the administered tracer and the target of interest, in the scanned object. PET studies are performed either dynamically or statically. Static PET

\footnotetext{
Received Jan. 21, 2010; revision accepted Mar. 26, 2010.

For correspondence or reprints contact: Pasha Razifar, GEMS PET Systems, Uppsala Applied Science Laboratory (UASL), GE Healthcare, Husbyborg, Uppsala 752 28, Sweden.

E-mail: pasha.razifar@ge.com

COPYRIGHT @ 2010 by the Society of Nuclear Medicine, Inc.
}

studies are often used in clinical applications such as oncology and neurology with already well-known tracers, such as ${ }^{18}$ F-FDG. Dynamic PET studies are often used in clinical applications such as neurology, cardiology, and oncology to explore the kinetic behavior of an administered tracer in the scanned object and to study the treatment effect.

In contrast to static imaging, dynamic PET studies depict the same volume within the scanned object but at different time points during the study, suggesting the possibility of exploring the physiologic interactions of the administered tracer in the scanned object as a function of time. Furthermore, dynamic PET studies can be used to explore the kinetic behaviors of new tracers or existing tracers in new applications (1).

However, independent of the type of study performed, PET data often have high levels of noise, which make it difficult to discern different areas in image volumes. A standard method for reducing noise and improving qualitative and quantitative estimation is to sum or average image volumes within a chosen time interval. The summation is usually performed over parts of the sequence in which the signal is proportionally higher. Furthermore, the signal often has a higher amplitude in the beginning of a scan, when the tracer has not yet bound to the target. This means that the signal in acquired image volumes is based on flow rather than binding, which is not the desired result. It is possible to improve the results by summing image volumes within later time frames of the scan; however, the level of noise will increase significantly, leading to a loss of quantitative information, especially when ${ }^{11} \mathrm{C}$-labeled tracers with a half-life of $20 \mathrm{~min}$ are used (2).

The level of noise in a PET dataset considerably affects the accuracy of quantitative results, especially with kinetic modeling methods, in which noise causes large uncertainty in the parameters used as the modeling assumptions. Because the parameters used in kinetic modeling are partly determined by the properties within a reference region, modeling methods are dependent on either accurate blood sampling or an appropriate choice of a reference region to be used as an input function. The aim of kinetic modeling is to increase differences between healthy and pathologic 
regions. One of the most well-known kinetic modeling methods is the so-called reference Patlak method (3). However, the reference Patlak method applied to an image domain does not consider optimization of the signal-tonoise ratio, and the parametric images that are obtained have high levels of noise. Wang et al. showed that the signal-to-noise ratio in parametric images could be improved by applying the Patlak method to a sinogram domain (4). Some other kinetic modeling methods are Logan plots (5), compartment modeling, factor analysis, and spectral analysis (6). Common to all kinetic modeling methods is low quality, especially when the data used are noisy or when the reference region contains any specific or nonspecific binding.

A recently introduced method, masked volumewise principal-component analysis (MVW-PCA), can be used to analyze data without making any modeling assumptions. Although quantitative information is lost when separate principal components are evaluated with this method, the diagnostic value of the resulting image is often better than that obtained with the other methods mentioned $(1,2)$. MVW-PCA has the ability to extract and separate uncorrelated signals representing different kinetic behaviors of the administered tracer. Consequently, the image volumes resulting from MVW-PCA are well suited for drawing regions of interest to be used as reference regions (7) or for creating time-activity curves (8).

Both static and dynamic PET data are usually stored in sinograms (9). In a static study, each slice in the scanned object is represented by a sinogram. In a dynamic study, every slice is represented by a set of sinograms, one for each time interval or frame. The sinograms contain information about the number of counts made by a detector pair, but the temporal resolution is limited to the frame to which the counts belong. The sinograms are reconstructed into images by use of a reconstruction algorithm. A drawback of the sinogram storing method is that the time protocol must be chosen before the data acquisition begins and cannot be changed after the scan. The list-mode (10) data storing option available in some newer PET cameras offers the opportunity to change dynamic time protocols after the PET scan. In the list mode, all detections made by
Sum image
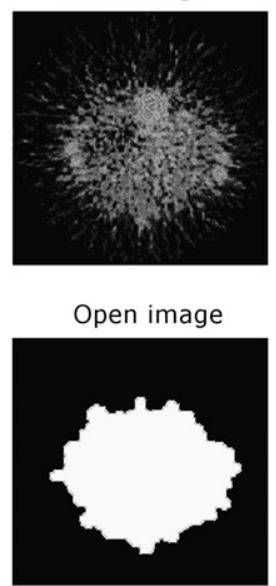

Threshold

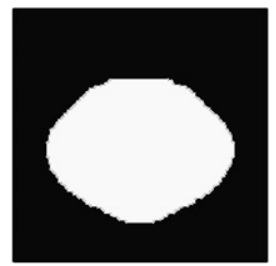

Threshold

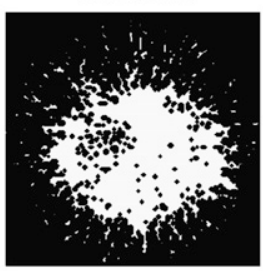

Symmetric

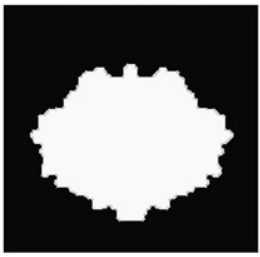

z - filter

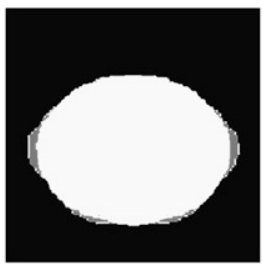

Fill holes

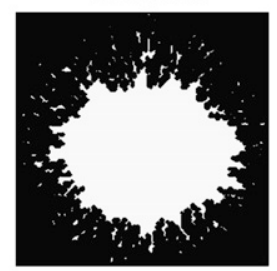

xy - filter

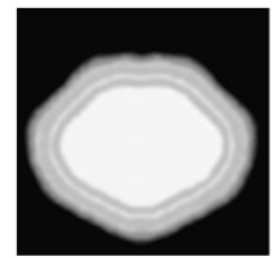

Threshold

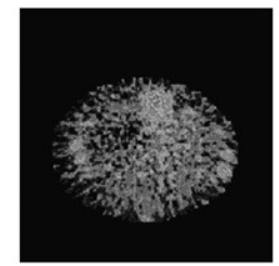

FIGURE 1. Step-by-step masking of PET series created in small-animal PET study of rat with ${ }^{18} \mathrm{~F}$ tracer. Results shown were obtained after masking steps for slice 30 of 61 .

the PET camera during the scan are stored in a list containing information about which detector has detected a photon and a time stamp with the precise time of the detection. This information makes it possible to construct sinograms after the acquisition is completed with any desired time protocol. This feature is important for the evaluation of multivariate image analysis methods for PET data because it is important for the methods used to be stable with respect to the choice of a time protocol. Without the list-mode storing option, it is difficult to analyze the effects of different time protocols with multivariate analysis methods because the same acquisition data are available only with one time protocol.

In the present study, the effects of using datasets with different time protocols as inputs to MVW-PCA were
FIGURE 2. Slice 31 of 61 from MVW$P C 1$ images of mouse data. Time protocols had frame lengths of $1 \mathrm{~min}(\mathrm{~A}$ and $\mathrm{E}), 2$ min (B and $F), 5$ min ( $C$ and $G)$, and 10 min $(D$ and $H)$. Spine is clearly visible in upper portions of images. There are no visible differences among time protocols. (A color version of this figure is available as a supplemental file online at http://tech.snmjournals.org.)
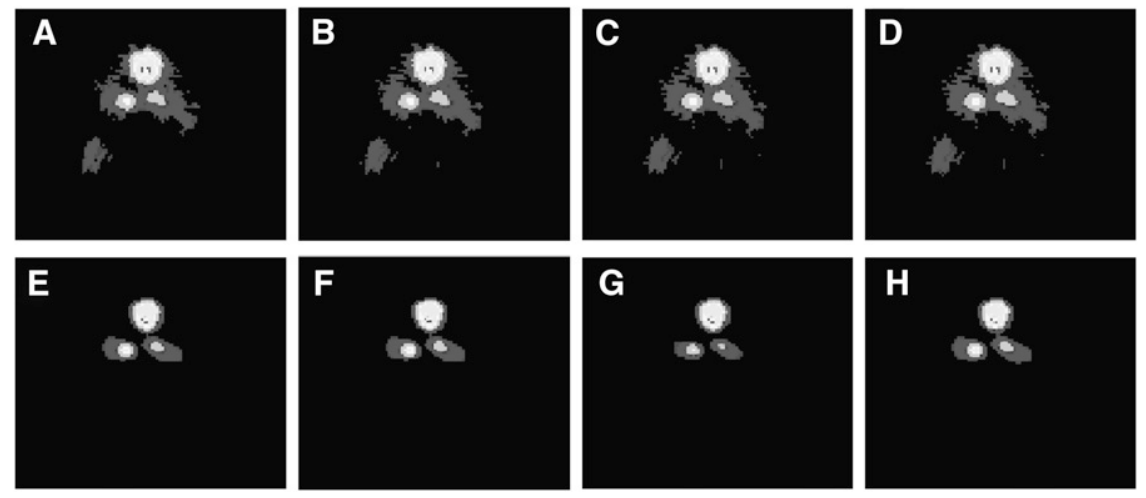
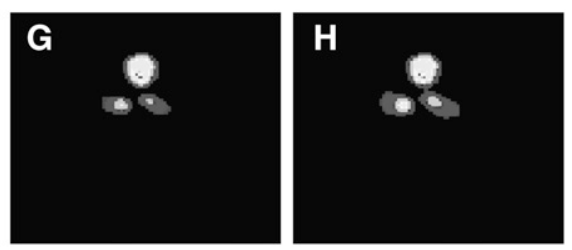


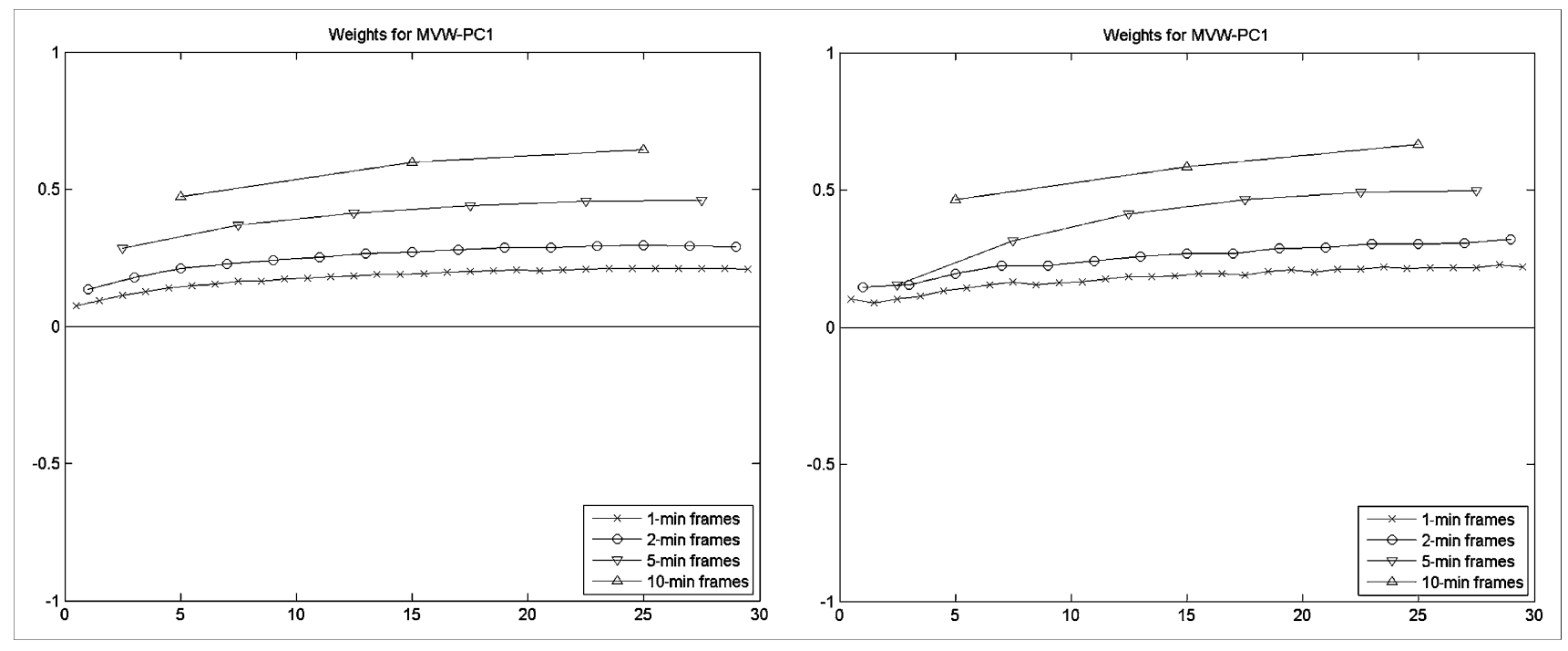

FIGURE 3. Weight factors for MVW-PC1 images of mouse data. (Left) Weight factors for FBP-reconstructed images. (Right) Weight factors for OSEM-reconstructed images. In both cases, MVW-PC1 is close to mean image.

studied with list-mode PET acquisition data from in vivo animal studies performed with a fluorine tracer. The aim of the study was to evaluate the performance of MVW-PCA in terms of signal extraction and separation as well as image quality with different time protocols in the input dataset.

\section{MATERIALS AND METHODS}

\section{Data Acquisition}

The animal study was performed with an eXplore VISTA dualring small-animal PET scanner (GE Healthcare). The unit contains 2 rings of Phoswich detector modules capable of performing 3-dimensional data acquisition with an axial field of view of 48 $\mathrm{mm}$ and an effective transaxial field of view of $67 \mathrm{~mm}$. The spatial resolution for reconstructions made with 3-dimensional orderedsubset expectation maximization (OSEM) is $0.8-1.0 \mathrm{~mm}$, and that when images are reconstructed with filtered backprojection (FBP) is $1.5-1.8 \mathrm{~mm}$ (11). Each time frame consisted of 61 transaxial slices with an image resolution of $175 \times 175$.

The data acquisition was performed dynamically with the list mode so that we could divide the dataset with various time protocols before reconstruction with either iterative or analytic approaches. The time protocols for the 30-frame, 15-frame, 6-frame, and 3-frame datasets were $30 \times 1 \mathrm{~min}, 15 \times 2 \mathrm{~min}, 6 \times$ $5 \mathrm{~min}$, and $3 \times 10 \mathrm{~min}$, respectively.

\section{Animal Studies}

Studies were performed on 4 C57 mice and 10 SpragueDawley rats. All animal studies were approved by the local ethics committee and performed in conjunction with the Guide for the Care and Use of Laboratory Animals (12).

Under anesthesia, an animal was placed in the prone position in the scanner gantry, and ${ }^{18} \mathrm{~F}$-fluoride $(\sim 10 \mathrm{MBq})$ was injected via the tail vein. This study was performed to explore possible mineralization in soft tissues when animals were treated with a substance of interest (an ongoing project). Image acquisition and radiotracer injection were started simultaneously, and a wholebody dynamic sequence (14 frames: $4 \times 30 \mathrm{~s}, 4 \times 60 \mathrm{~s}, 2 \times 120 \mathrm{~s}$, $2 \times 300 \mathrm{~s}$, and $2 \times 1,200 \mathrm{~s}$ ) was acquired over $120 \mathrm{~min}$.

\section{PCA}

PCA is an unsupervised method that linearly transforms a given set of multivariate variables to a new set of variables. The transformation matrix is chosen in such a way that base vectors lie in the direction of maximum variance among all possible linear combinations of the variables in the multivariate dataset. The resulting
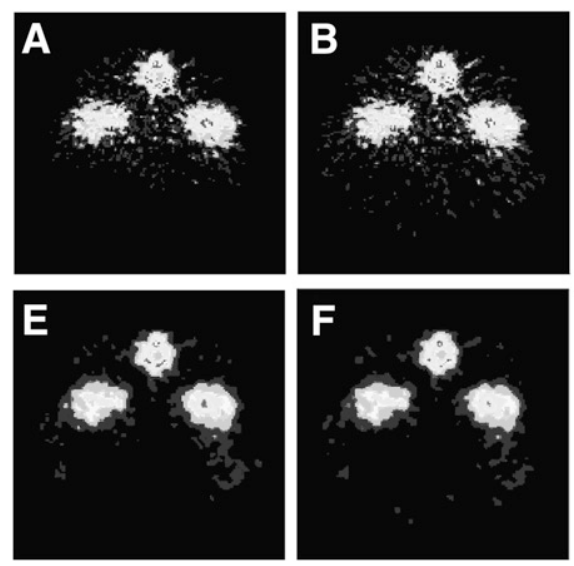
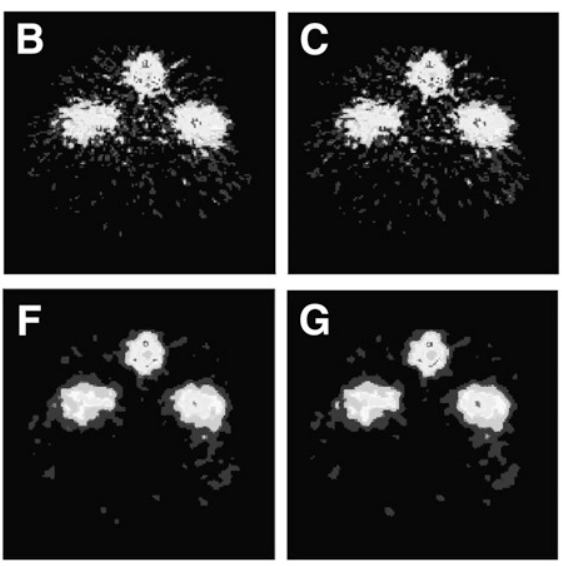

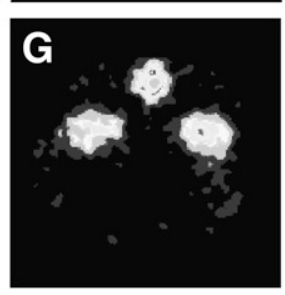

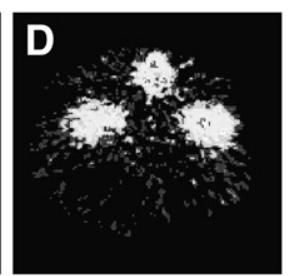

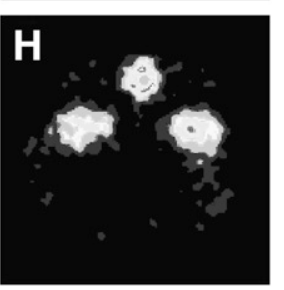

FIGURE 4. Slice 31 of 61 from MVWPC1 images of data acquired in rat study. Time protocols had frame lengths of 1 $\min (A$ and $E), 2$ min (B and $F), 5$ min (C and $G$ ), and 10 min (D and $H)$. Spine is clearly visible in upper portions of images. Kidneys are visible in left and right portions of images. There are almost no visible differences among time protocols. (A color version of this figure is available as a supplemental file online at http:// tech.snmjournals.org.) 


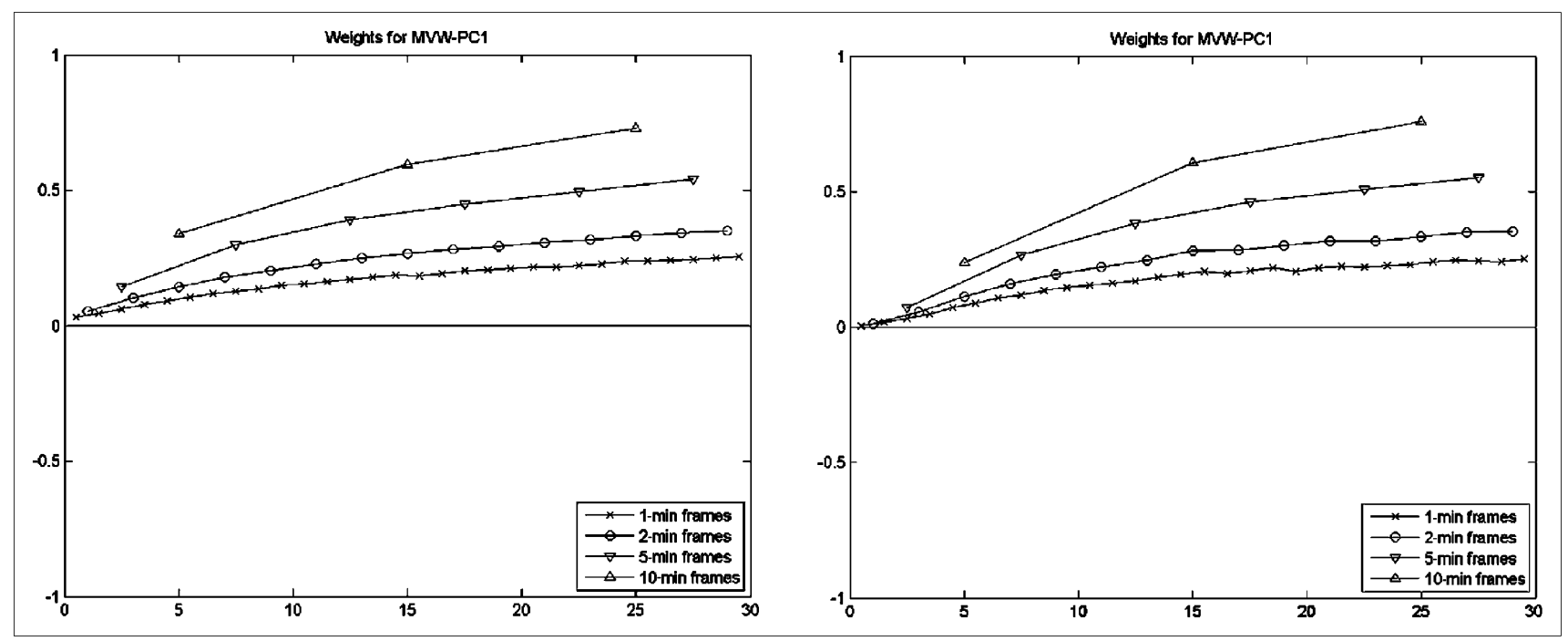

FIGURE 5. Weight factors for MVW-PC1 images of rat data. (Left) Weight factors for FBP-reconstructed images. (Right) Weight factors for OSEM-reconstructed images. In both cases, MVW-PC1 assigns higher weights to later frames, which typically contain less noise than earlier frames.

variables yield maximum variance among all possible linear combinations of the dataset under the constraint that they should be orthogonal to all of the previously chosen variables.

Because PCA cannot separate variance due to a signal from variance due to noise, prenormalization must be applied before PCA. Prenormalization transforms the dataset to a new dataset from which the signal can be extracted more easily. The noise in raw PET data is usually considered to have a Poisson distribution, but after reconstruction of image sequences from raw data by a method such as FBP, the noise can be considered to have an approximate gaussian distribution. The prenormalization method (background noise prenormalization) was performed by dividing the value of each pixel in the image by the standard deviation of the background in the image. Because the background pixel values in the OSEM-reconstructed image were 0 , they could not be used to estimate the noise in the image. Instead, a new approach (an ongoing project), high-order principal-component analysis, was used. This approach is based on an estimation of the noise with information obtained from higher-order principal components.

\section{MVW-PCA}

In MVW-PCA, which was developed by Razifar et al. $(1,13)$, the entire dynamic image volume is used as input data for PCA.
To avoid inconsistencies among different slices of the object, the entire volume is treated as one observation. This approach is logical because the scanned object is not built up in slices. Moreover, the results of PCA should depend on image data from the scanned object and not the volume around the object; therefore, the object is masked and extracted. Masking can be done with transmission or CT data if such data are available.

Because no CT or attenuation data were available, an automatic masking technique was developed. Masking of the reconstructed PET data was performed by first summing all of the frames. After summation was performed, a threshold at the mean value in the slice was used to create an initial separation of the object from the background. However, because of the high level of noise in the reconstructed PET data, especially when the images were reconstructed with FBP, several steps were needed to create a highquality mask. First, the holes were filled with morphologic operations. Next, a standard image opening was performed with erosion and dilation of the image. A scanned object often is approximately symmetric in the vertical direction ( $x$-direction), but tracer binding is sometimes not symmetric, yielding a nonsymmetric mask. To correct this problem, the mask can be made symmetric by adding the mask to a mirrored version of itself. The result of these operations often is a jagged mask. Filtering with
FIGURE 6. Slice 31 of 61 from MVWPC2 images of data acquired in mouse study. Time protocols had frame lengths of $1 \mathrm{~min}$ ( $A$ and $E$ ), 2 min (B and $F$ ), 5 min (C and $G)$, and 10 min (D and $H$ ). Kidneys and urinary tract are clearly visible in images. Differences among time protocols are slight, especially for comparison of spine in upper portions of $A$ and D. Spine in $G$ has slightly higher amplitude than spine in $E, F$, and $H$. (A color version of this figure is available as a supplemental file online at http:// tech.snmjournals.org.)
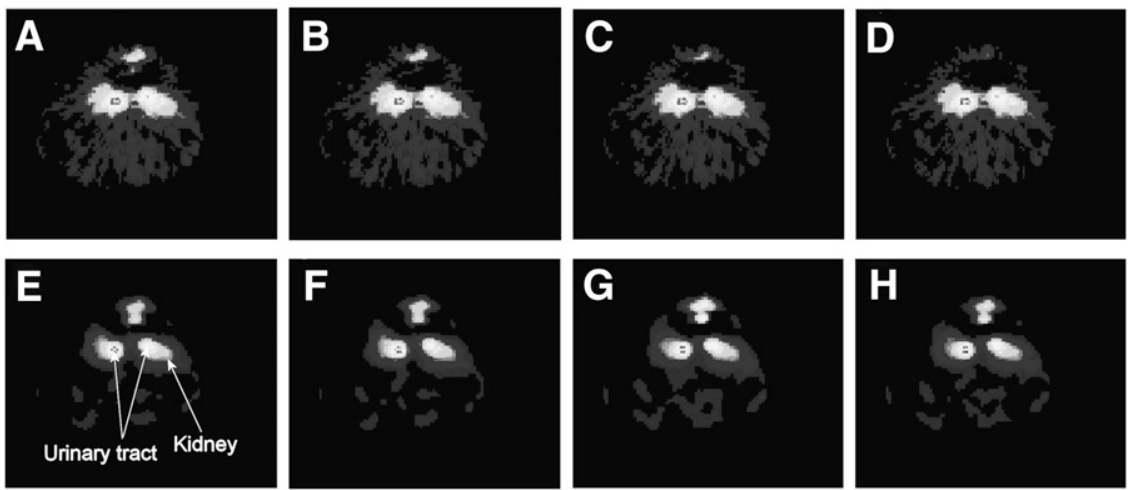
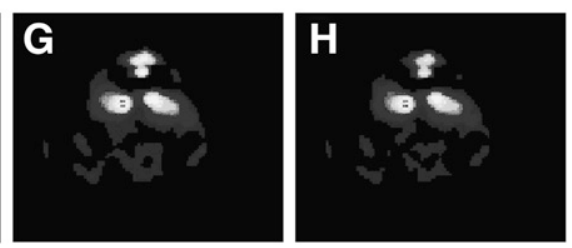
a large mean filter and applying a threshold reduce this problem. The threshold level can be varied to provide higher or lower sensitivity to small details. Furthermore, there are often inconsistencies between slices. This problem is solved by correlating the slices by 3-dimensional convolution of the mask with a rod-shaped kernel aligned in the axial direction (z-direction) and again applying a threshold. The threshold level can be varied like the previous threshold level to provide higher or lower sensitivity to small details. The result of each step in the masking algorithm is shown in Figure 1. Altogether, the masking takes approximately $1 \mathrm{~s}$ per slice.

For masking of image series reconstructed with OSEM, some of the smoothing steps described earlier are not strictly necessary but sometimes can improve the results. Otherwise, the results of this masking method applied to images reconstructed with OSEM and images reconstructed with FBP are similar.

\section{Data Analysis}

The datasets from the animal studies were analyzed with MVW-PCA. The data analysis was performed in a few steps; the acquired PET data were reconstructed from the list mode with FBP or OSEM and 4 time protocols. The images were then masked with the masking technique described earlier. After masking was performed, the images from FBP were prenormalized with the background SD normalization technique described earlier. Finally, PCA was performed on the masked (and, for images from FBP, normalized) data, with the entire masked object from different frames being treated as one observation. After PCA was performed, the result was backprojected to the mask.

\section{RESULTS}

All images had $175 \times 175$ pixels of original data; the images were cropped to better illustrate the results. Of the images shown in Figures 2-9, those in Figures 2, 4, 6, and 8 were reconstructed with FBP (A-D in those figures) and with OSEM (E-H in those figures).
The upper portions of the MVW-PC1 images from the mouse data shown in Figure 2 clearly reveal the spine; the kidneys are visible below the spine but have a much lower amplitude. These data represent nearly mean behavior, as indicated by the weight factors shown in Figure 3. There is almost no visible difference in data obtained with the various time protocols, and the behavior with OSEM is similar to that with FBP.

The upper portions of the MVW-PC1 images from the rat data shown in Figure 4 clearly reveal the spine; the kidneys are also visible and have a high amplitude. These data represent mean behavior, with an emphasis on the signal in the later frames, as indicated by the weight factors shown in Figure 5. There is almost no visible difference in data obtained with the various time protocols, and the behavior with OSEM is similar to that with FBP.

The MVW-PC2 images from the mouse data clearly show the kidneys, whereas the spine has a low amplitude. There is a small difference between OSEM, with which some of the spine is still visible, and FBP, with which the spine has an amplitude of almost 0 (Fig. 6). This difference is also visible in the weight factors shown in Figure 7.

The MVW-PC2 images from the rat data clearly show the kidneys, whereas the spine has an amplitude of almost 0 . There is almost no visible difference between FBP and OSEM (Fig. 8), as indicated by the weight factors shown in Figure 9.

The different areas visible in both MVW-PC1 and MVW-PC2 images (Fig. 10) were used to calculate timeactivity curves (Fig. 11). The data clearly show the separation of different kinetic behaviors.

The images resulting from MVW-PCA clearly show the separation of tracer kinetics in the spine into MVW-PC1 images and in the soft tissues of the kidneys and urinary
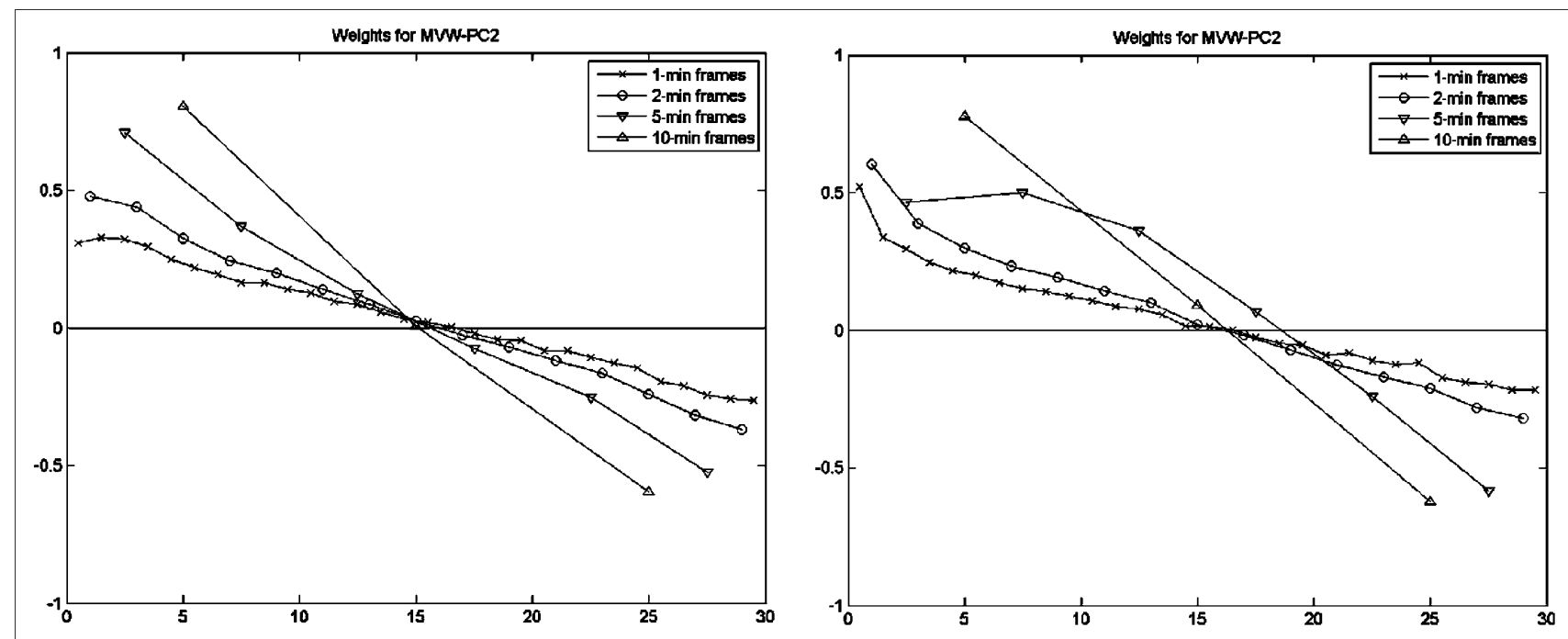

FIGURE 7. Weight factors for MVW-PC2 images of mouse data. (Left) Weight factors for images reconstructed with FBP. (Right) Weight factors for images reconstructed with OSEM. In both cases, MVW-PC2 assigns higher weights to earlier frames, which show higher signal intensity in kidneys, and negative weights in second half of scan, in which kidneys show little activity. 
FIGURE 8. Slice 31 of 61 from MVWPC2 images of data acquired in rat study. Time protocols had frame lengths of $1 \mathrm{~min}(A$ and $E), 2$ min ( $B$ and $F$ ), 5 min ( $C$ and $G$ ), and 10 min (D and $H)$. Kidneys and urinary tract are clearly visible in middle portions of images. There are small but visible differences among OSEM-reconstructed images $(E-H)$. (A color version of this figure is available as a supplemental file online at http:// tech.snmjournals.org.)
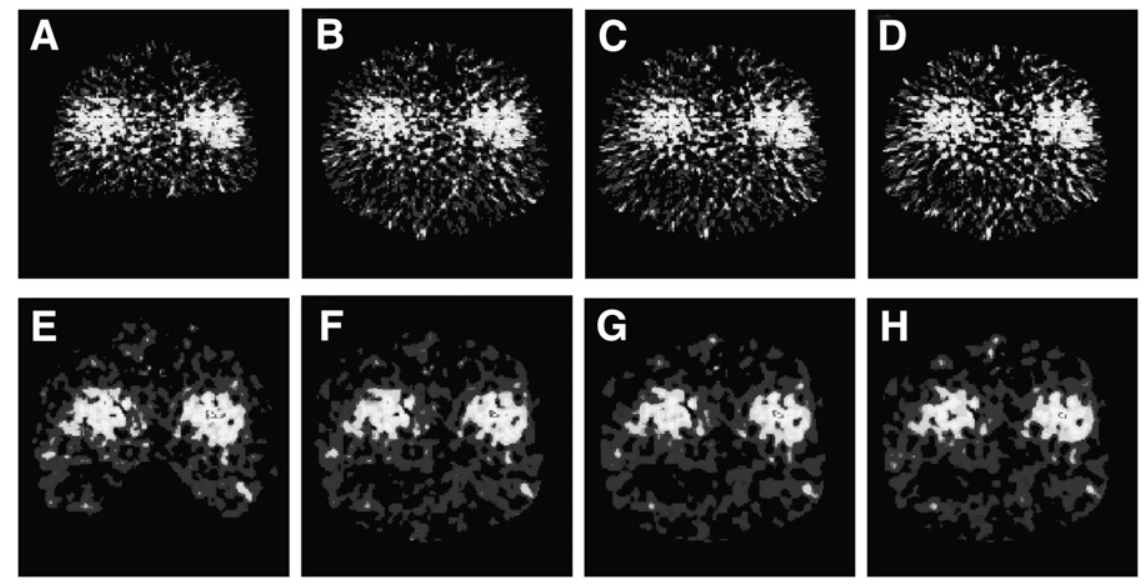

tract into MVW-PC2 images. We fused MVW-PC1 and MVW-PC2 volumes and illustrated the results using maximum-intensity projection (MIP), showing organs with different kinetic behaviors in different colors (Supplemental Video 1) (supplemental materials are available online only at http://tech.snmjournals.org). There are almost no visible differences in the images, regardless of the frame lengths chosen.

\section{DISCUSSION}

To provide useful results from any analysis method used for PET data, the method should not depend on parameters specified by the user, such as the time protocol chosen for a dynamic PET scan. The results obtained with the analysis method should depend as much as possible on the signal; little or no variation should depend on other parameters. With kinetic modeling methods, the results are markedly dependent on the proper choice of the modeling approach on the basis of kinetic behavior and predefined parameters. MVW-PCA does not have modeling parameters like those used in kinetic modeling or summation methods, but the results may depend on the time protocol chosen for the PET scan.

In the present study, the performance of MVW-PCA was studied with different time protocols and reconstruction algorithms. The aim of the study was to investigate the stability of the method with respect to the time protocols chosen and the extraction and separation of signals with different kinetic behaviors.

When dynamic PET data are analyzed with MVW-PCA, the resulting images are weighted means of the different frames. However, MVW-PCA uses no information about the difference in frame lengths within the dataset; therefore,

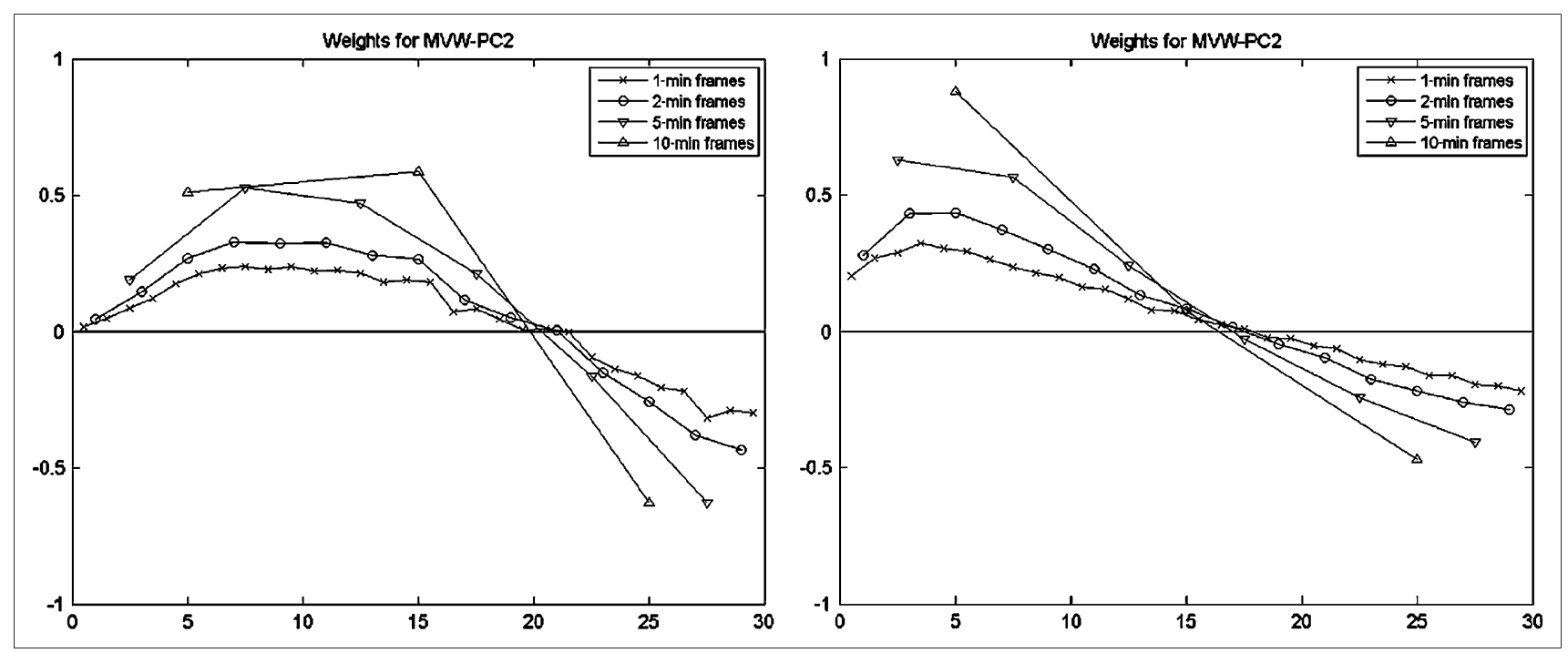

FIGURE 9. Weight factors for MVW-PC2 images of rat data. (Left) Weight factors for images reconstructed with FBP. (Right) Weight factors for images reconstructed with OSEM. In both cases, MVW-PC2 assigns higher weights to first half of frames, which contain higher signal intensity in kidneys, and negative weights in last part of scan, in which kidneys have little activity. There are differences between FBP and OSEM in shapes of weight factors, especially in beginning of scan, in which weight factor for OSEMreconstructed data (right) is close to 0 in first part of scan. 

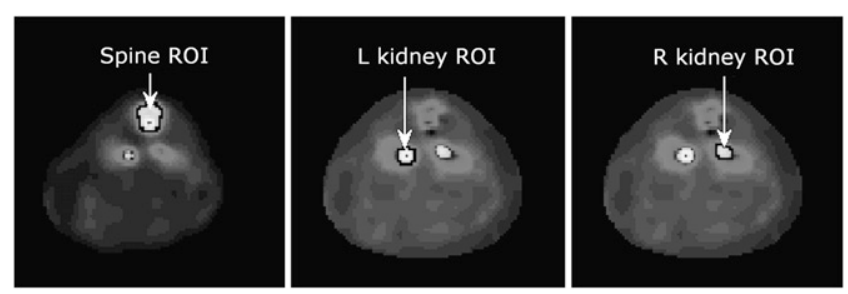

FIGURE 10. Regions of interest (ROls) used to calculate timeactivity curves shown in Figure 11. ROls are drawn on MVW-PC1 image for spine and on MVW-PC2 images for kidneys. (A color version of this figure is available as a supplemental file online at http://tech.snmjournals.org.)

the individual frames are considered equally important when the covariance of the input data is calculated. Therefore, the use of short frame lengths in the beginning of the scan so as to discern rapid kinetics, such as those seen in the blood vessels in a human brain PET scan, not only will produce the effect of a high sampling frequency but also will result in the beginning of the scan having more impact when MVW-PCA is performed. In the present study, the frame lengths used for image reconstruction were equal throughout the entire scan so as to avoid making any assumptions about different tracer kinetics and thereby emphasizing any part of the dataset. However, for most clinical and preclinical PET scans, frame lengths are usually shorter at the beginning of a scan to compensate for the decay of the radionuclide.

The images resulting from MVW-PCA show that the use of different frame lengths has little effect on these images. The difference seen among the weight factors corresponding to the different time protocols is mostly a scaling factor introduced because of the different numbers of frames in the datasets. This situation is due to the eigenvectors of the covariance matrix having unit length. For images resulting from MVW-PCA, the scaling factor is not visible because the images have been scaled to the same amplitude interval.

The lack of difference in the results from the datasets with different time protocols can be explained by the timeactivity curves for the regions with the highest activity in the dataset shown in Figure 11. The kinetics in the spine indicate that the tracer accumulates slowly but remains throughout the scan. The kinetics in the kidneys and urinary tract indicate a high tracer concentration immediately at the beginning of the scan and then a rapid decline to a low concentration for the remainder of the scan. These 2 kinetic behaviors are easily separated with only a few frames.

Studying the weight factors and comparing them to the time-activity curves for the different regions can explain the results for the MVW-PC2 images, in which the spine has an amplitude close to 0 . The early frames show a high amplitude in the kidneys but also some amplitude in the spine. The fact that the spine is nearly removed from the MVW-PC2 images is explained by the negative weights in the later part of the scan, in which the spine has high activity but there is nearly no activity in the kidneys.

The dataset with only 3 frames was sufficient to separate the spine and kidneys even though the sampling frequency was too low to describe the rapid kinetics in the kidneys with any precision. Additionally, the difference between images resulting from MVW-PCA with 30 frames and images resulting from MVW-PCA with 3 frames was small. These findings lead to the conclusion that MVW-PCA is a stable method that depends more on the signal than on the time protocol used for image reconstruction.

\section{CONCLUSION}

MVW-PCA can efficiently separate different kinetic behaviors and provide images in which areas with different tracer uptake kinetics are easier to discern. Moreover, MVW-PCA is

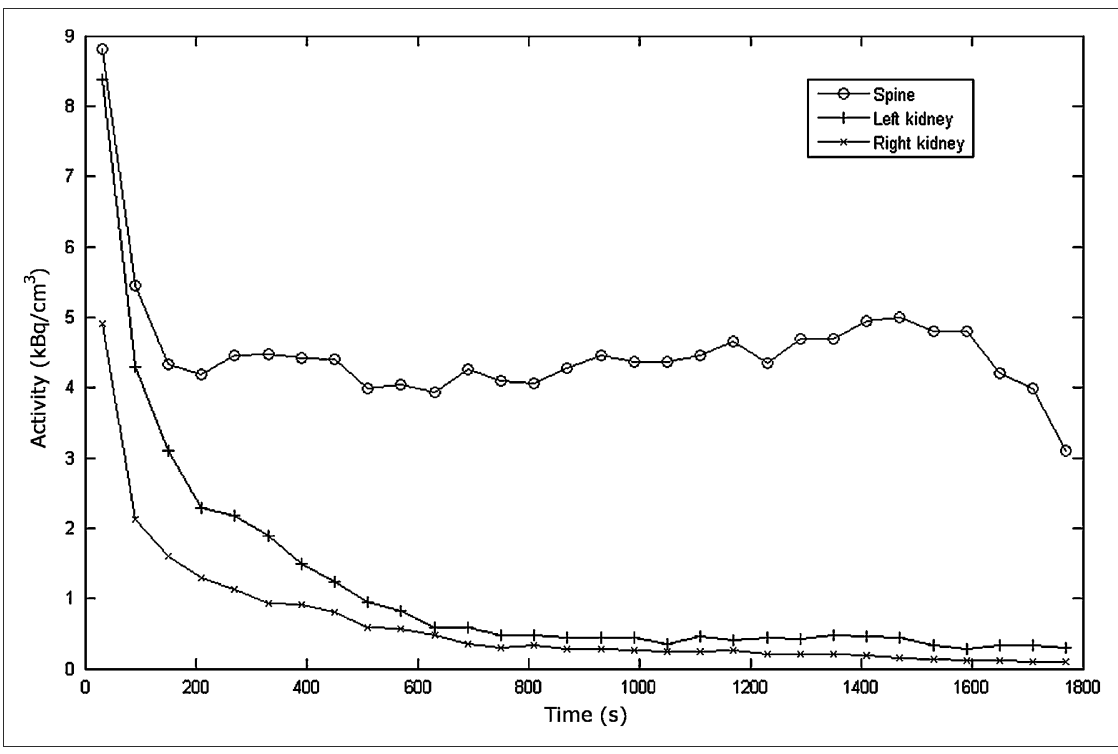

FIGURE 11. Time-activity curves for spine and left and right kidneys. Activity data were collected from 1-min frame of original dataset obtained from scanned mouse and reconstructed with FBP. 
a stable technique in the sense that the time protocol chosen has a small impact on image quality but not on separation.

\section{REFERENCES}

1. Razifar P, Axelsson J, Schneider H, Långström B, Bengtsson E, Bergström M. A new application of pre-normalized principal component analysis for improvement of image quality and clinical diagnosis in human brain PET studies: clinical brain studies using $\left[{ }^{11} \mathrm{C}\right]-\mathrm{GR} 205171,\left[{ }^{11} \mathrm{C}\right]-\mathrm{L}$-deuterium-deprenyl, $\left[{ }^{11} \mathrm{C}\right]-$ 5-hydroxy-L-tryptophan, $\left[{ }^{11} \mathrm{C}\right]-\mathrm{L}-\mathrm{DOPA}$ and Pittsburgh compound-B. Neuroimage. 2006;33:588-598.

2. Razifar P, Engler H, Blomquist G, et al. Principal component analysis with prenormalization improves the signal-to-noise ratio and image quality in positron emission tomography studies of amyloid deposits in Alzheimer's disease. Phys Med Biol. 2009;54:3595-3612.

3. Patlak CS, Blasberg RG, Fenstermacher JD. Graphical evaluation of blood-tobrain transfer constants from multiple-time uptake data. J Cereb Blood Flow Metab. 1983;3:1-7.

4. Wang G, Fu L, Qi J. Maximum a posteriori reconstruction of the Patlak parametric image from sinograms in dynamic PET. Phys Med Biol. 2008; 53:593-604.
5. Logan J. Graphical analysis of PET data applied to reversible and irreversible tracers. Nucl Med Biol. 2000;27:661-670.

6. Cunningham VJ, Jones T. Spectral analysis of dynamic PET studies. J Cereb Blood Flow Metab. 1993;13:15-23.

7. Razifar P, Engler H, Ringheim A, Estrada S, Wall A, Långström B. An automated method for delineating a reference region using masked volumewise principalcomponent analysis in ${ }^{11}$ C-PIB PET. J Nucl Med Technol. 2009;37:38-44.

8. Razifar P, Hennings $\mathrm{J}$, Monazzam A, Hellman $\mathrm{P}$, Långström $\mathrm{B}$, Sundin A. Masked volume wise principal component analysis of small adrenocortical tumours in dynamic $\left[{ }^{11} \mathrm{C}\right]$-metomidate positron emission tomography. BMC Med Imaging. 2009;9:6.

9. Fahey FH. Data acquisition in PET imaging. J Nucl Med Technol. 2002;30:39-49.

10. Nichols TE, Qi J, Asma E, Lehay RM. Spatiotemporal reconstruction of list mode PET data. IEEE Trans Med Imaging. 2002;21:396-404.

11. Wang Y, Seidel J, Tsui BMW, Vaquero JJ, Pomper MG. Performance evaluation of the GE Healthcare eXplore VISTA dual-ring small-animal PET scanner. $J$ Nucl Med. 2006;47:1891-1900.

12. Guide for the Care and Use of Laboratory Animals. Washington, DC: National Academy Press; 1996.

13. Razifar P, Axelsson J, Schneider H, Långström B, Bengtsson E, Bergström M. Volume-wise application of principal component analysis on masked dynamic PET data in sinogram domain. IEEE Trans Nucl Sci. 2006;53:27592768. 